\title{
Relationship between Seismic Activity and Variations in the Earth's Rotation Angular Velocity
}

\author{
Elena Sasorova ${ }^{1} \&$ Boris Levin $^{1,2}$ \\ ${ }^{1}$ Shirshov Institute of Oceanology RAS, Moscow, Russian Federation \\ ${ }^{2}$ Institute of Marine Geology and Geophysics FEB RAS, Yuzhno-Sakhalinsk, Russian Federation \\ Correspondence: Elena Sasorova, Shirshov Institute of Oceanology RAS, 36 Nakhimovskii prospect, 117208, \\ Moscow, Russian Federation. Tel: 7-499-1248713. E-mail: sasorova_lena@mail.ru
}

Received: February 26, $2018 \quad$ Accepted: March 2, $2018 \quad$ Online Published: May 7, 2018

doi:10.5539/jgg.v10n2p43 URL: https://dx.doi.org/10.5539/jgg.v10n2p43

\begin{abstract}
The Earth's seismic activity (SA) demonstrates a distinct unevenness both in space and in time. The periods of intensification of seismic activity are followed by periods of its decline. In this work, an attempt was first made to determine the effect of low-frequency components of the variations in the angular velocity of the Earth's rotation (AVER) on the dynamics of its seismic activity (for 1720 - 2017). Analysis of the time series of the density of seismic events and variations in the Earth's rotation velocity of about 300 years shows that each stage of reducing the angular velocity of rotation (braking) is accompanied by an increase in the density of seismic events, and the stages of increasing the angular velocity of rotation (acceleration) are accompanied by a decrease in the density of events. At present, the Earth is entering a new phase of deceleration (since 2005), which in recent years has already led to an increase in the global seismic activity.
\end{abstract}

Keywords: global seismicity, magnitude, angular velocity of the Earth, braking stage, acceleration stage

\section{Introduction}

Seismic activity of the Earth demonstrates a distinct unevenness both in space and in time. The periods of intensification of the seismic activity (SA) are followed by periods of its decline. According to various estimates (Levin\&Sasorova, 2015a; Levin \& Sasorova, 2015b; Lutikov\&Rogozhin, 2014) for strong earthquakes (ST), these cycles continue for several decades, cycles of about 30, 55 and 70 years have been observed. In these works series of seismic events with a duration of more than 100 years with $\mathrm{M}>=7$ or 7.5 were considered.

Attempts to discover the connection between global seismic activity and the features of the Earth's rotation have been undertaken repeatedly and earlier. But mainly earthquake catalogs, no more than 50 years old (since 1964), and time series on variations of the angular velocity of the Earth from the generally accessible base of the International Earth Rotation and Reference System Service (IERS) have been used for 40-50 years (available since 1962). However, the analysis of short time series does not make it possible to correctly identify the periodicity in the SA and in changes in the Earth's rotation velocity of more than 10-12 years. For the analysis of processes with periodicity of several decades, it is necessary to obtain long series of reliable observational data (at least 100 years) for both seismic events and for variations in the Earth's rotation velocity.

The objective of this work is to analyze the spatial-temporal distributions of the seismic events and relationship between the global SA and variations in the angular velocity of the Earth's rotation for the period from 1720 to 2017. Particular attention will be focused to the time period from 2005 to 2017, when our planet again entered the stage of reducing the angular velocity of rotation (into a new stage of braking).

\section{Selection of the Seismic Data}

To form a working catalog of strong EQ's (with $\mathrm{M}>=7.5$ ) for the period from 1720 to 2016 two subsets of the world catalog National Earthquake Information Center of the US Geological Survey (NEIC USGS) has been used. The first one is the catalog from 1973 to 2017, and the second one is the catalog of significant earthquakes on Earth from $2150 \mathrm{BC}$ to 1984 . Since the end of the XIX century earthquakes with $\mathrm{Ms} \geq 7.5$ can be considered representative. To complete the list of strong events for the period from 1720 to 1895, other sources were also used (for example, the Historical Tsunami Database for the World Ocean (HTDB/WLD)) 
Figure 1 shows the distribution of strong earthquakes from 1720 to 2017 with magnitude $M>=7.5$ over ten-year intervals for the entire Earth. With the beginning of the era of instrumental observations (1890), the number of registered seismic events sharply increases.

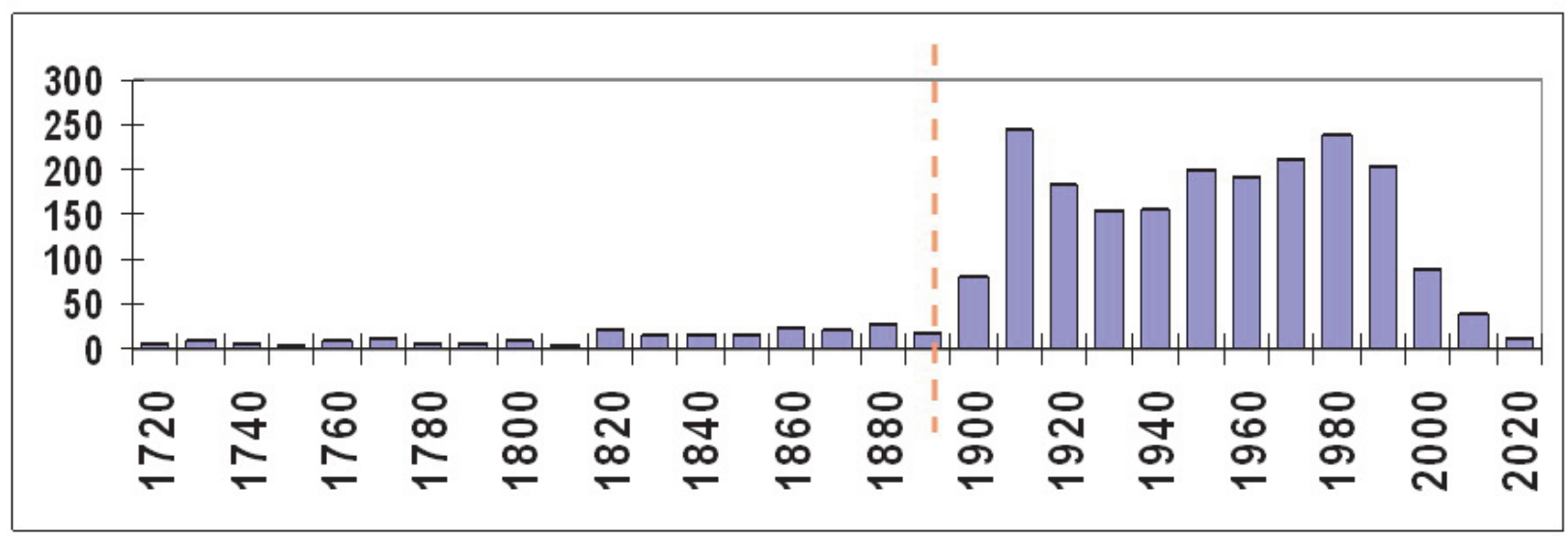

Figure 1. Distribution of the EQ's from 1720 to 2017 for ten-year intervals. The horizontal axis is the upper boundary of the ten-year intervals; the vertical axis is the number of events recorded in each interval. Red dashed line is the beginning of instrumental observations

Therefore, a comparative analysis of the SA and variations in the AVER at intervals of 1895-2017 and 1720 1895 was carried out separately. To prepare the working versions of the EQ's catalog, several procedures were conducted: few stages of preliminary treatment (exclusion of double events, distinguishing and correction of records with empty entries), standardization of magnitude scales, and removal of aftershock sequences.

Further to analyze the distributions of seismic events in time the entire observation interval was divided into five-year intervals, and the total number of events in each five-year interval was considered. The distributions of strong earthquakes $(M \geq 7.5)$ at 5-year intervals for the period 1720-1895 are shown in Figure 2a (total number of the EQ's - 92) and for the period 1895-2017 inFigure2b (total number of the events - 638).

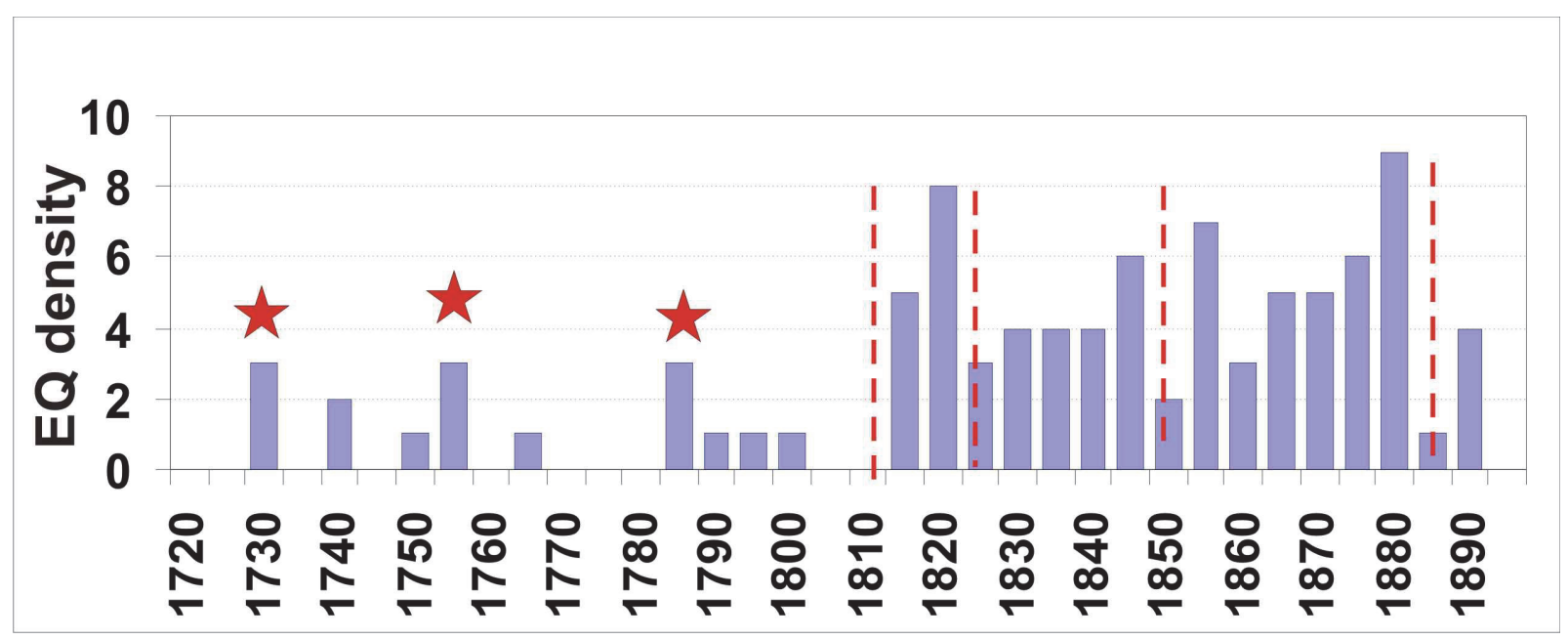

Figure 2a. Distribution of strong earthquakes $(M \geq 7.5)$ at 5-year intervals for the period 1720-1890. Red dotted vertical lines denote the local minima of the SA, and red asterisks, are indicate on catastrophic EQ. The vertical axis is the number of events recorded in each five-year interval 


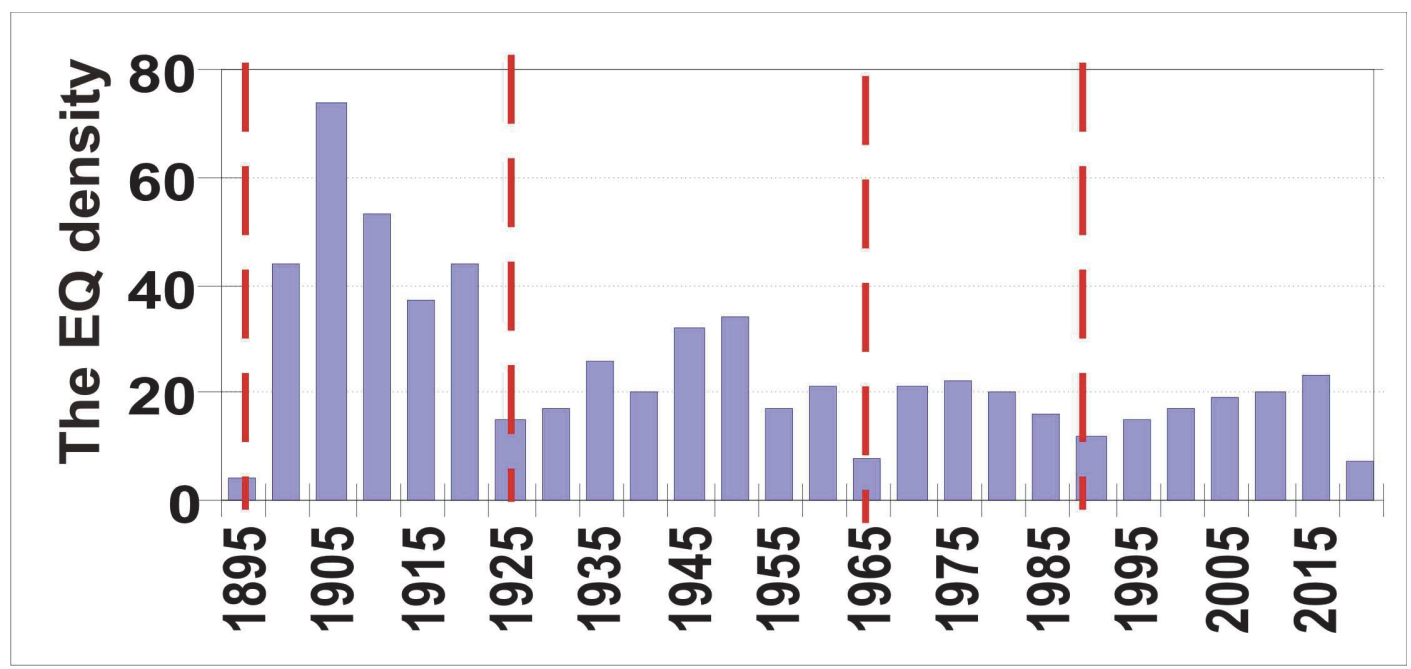

Figure 2b. Distribution of strong earthquakes $(M \geq 7.5)$ at 5-year intervals for the period 1895-2017. Red dotted vertical lines indicate the local minima of the SA. The vertical axis is the number of EQ's in each five-year interval

It should be noted that seismic events for the period from 1720 to 1895 were cataloged mainly on macro seismic data. The number of seismic events presented in the interval $1720-1810$ is equal to 16 . The maxima of the SA during this period, marked in Figure 2a by red asterisks, are associated with catastrophic EQ (Lisbon, 1755; South America, 1730 and Mexico, 1784; etc.) and they coincide in time with the stages of deceleration of the Earth. Since 1810 the number of recorded events had already increased in several times (to 76, an average of 4.75 events per 5-year interval). The data of the EQ catalogs from 1720 to 1810 can not be regarded as representative therefore the comparison of the density of seismic events and the variations of the Vn values were performed below only for the period from 1815 to 1890 .There may be observed a clearly expressed periodicity of the decrease and increase in the SA on both fragments of the figure.

\section{Selection of Data on Variations in the Earth's Rotation Rate}

Two data sources were adapted to analyze rotational velocity variations. This is freely available data of the agency IERS, daily observations of Length of day (LOD) from 1962 to 2017, collected by us in the SCAT catalog and tables presented in (McCarthy\&Babcock, 1986; Morrison, 1973). The first source collected data for the period 1720-1984 with an observation frequency of once every six months (collected in the catalog LCAT) .The catalogs have a period of overlap (1962-1984). In the considered sources, the values of LOD, defined as the difference in the period values $(\mathrm{Pz}-\mathrm{Pa})$, where $\mathrm{Pa}$ is the period of the astronomical day $(86400 \mathrm{sec})$ and $\mathrm{Pz}$ - the observed period of the Earth days ( $\mathrm{Pz}$ and $\mathrm{Pa}$ measured in millisecond) are presented. The relative variation of the angular velocity is defined as the relative change in the angular velocity, expressed in terms of their periods: $v=(\omega-\Omega) / \Omega \approx-(\mathrm{Pz}-\mathrm{Pa}) / \mathrm{Pa}$. The relative dimensionless value of the angular velocity variation is estimated as $v \approx 10^{-8}$ (Sidorenkov, 2002).

The LCAT catalog was then extended by 30 years, by adding values from 1984 to 2017 . The added values were obtained by averaging daily observations on each half-year interval (from 1962 to the present). Both catalogs have a common (overlapping) observation period from 1962 to 1984. A comparison of two time series for this period: the LCAT and the averaged series over the semiannual intervals of the SCAT showed that the average relative error does not exceed $5 \%$. 


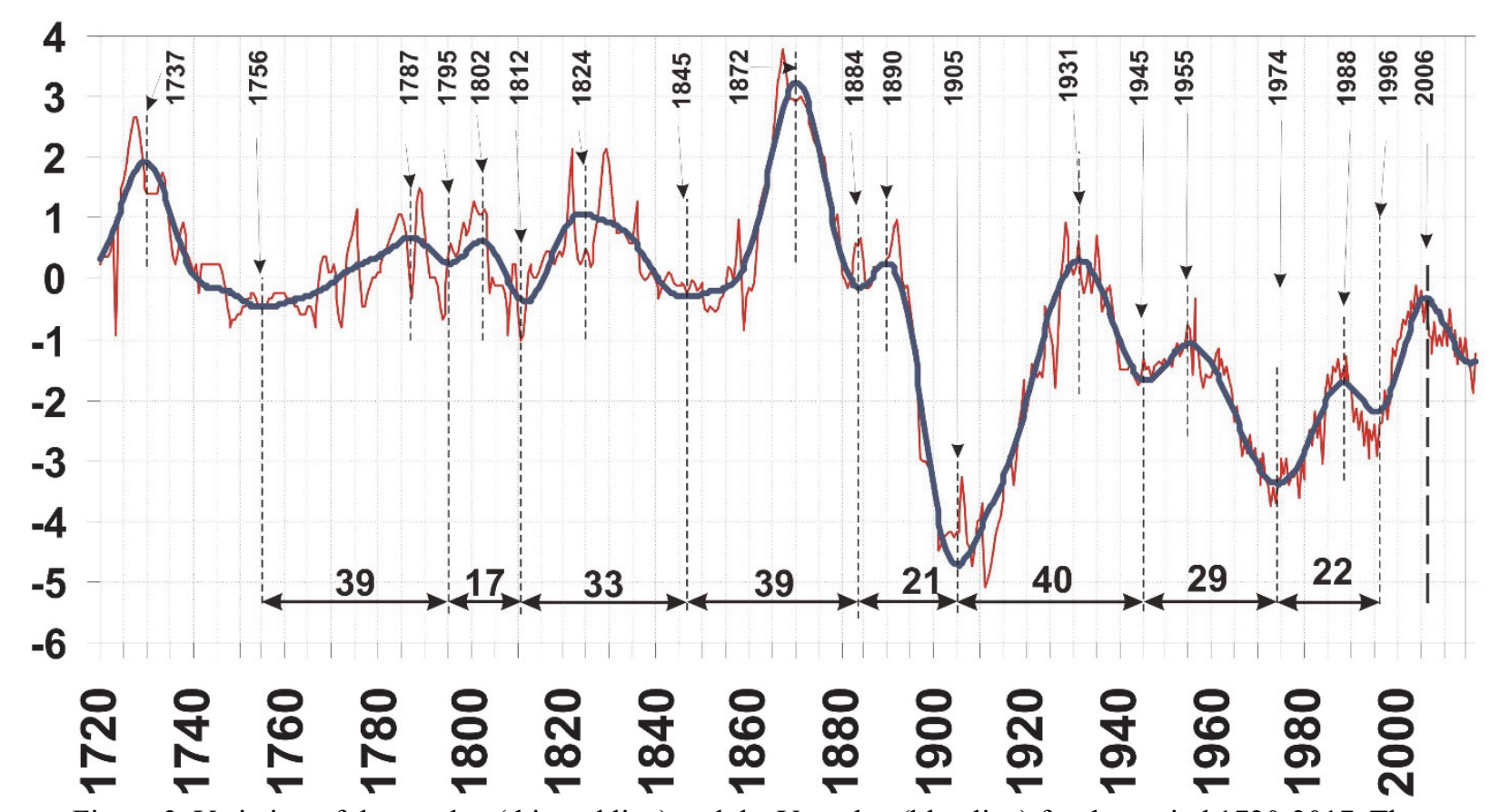

Figure 3. Variation of the $v$ value (thin red line) and the Vn value (blue line) for the period 1720-2017. The vertical axis indicate of the values $v^{*} 10^{8}$ and $\mathrm{Vn} * 10^{8}$. Black thin dashed vertical lines mark local extremes of the $\mathrm{Vn}$. In the upper part of the figure locate the times of appearance of each local extremes are indicated. The black thick dotted vertical line marks the beginning of the stage of the last period of Earth's deceleration. In the lower part of the figure, there are time intervals in years between two neighboring local minima $\mathrm{Vn}$.

The resulting combined catalog (JCAT) contains observations from 1720 to 2017 (297 years, 594 observations). The spectra for the time series from the JCAT and the SCAT catalogs were calculated in (Levin \&Sasorova, 2015b). The characteristic periods for the JCAT are 62 years, 32 years and 23 years. Therefore, further, we consider the total influence of all low-frequency components on the periodicity of the SA. The characteristic periods for the SCAT are one year, half a year, 28 and 14 days.

The time series Vn obtained as a result of filtering the values of $v$ from the combined JCAT catalog is shown in Figure 3 (extracted all periods more than 19 years). Figure 3 shows the prolonged periods (decades) of the significant increase $(1723-1737,1853-1878,1914-1939)$ and reducing $(1737-1764,1878-1892,1898-1914)$ of the $\mathrm{Vn}$ and relatively small variations around the certain level. The greatest range of local changes in Vn was observed in the period from 1872 to 1905 (from 3.5 to -4.5 relative units).

The time series obtained as a result of low-frequency filtering the $v$ values from the SCAT catalog (Vns) is shown in Figure $4 \mathrm{~b}$ (extracted all periods more than 2 years). All components of SCAT with periods of one year, 6 months, 14 and 28 days were excluded from this time series. Figures $4 \mathrm{a}$ and $4 \mathrm{~b}$ show low-frequency components for the JCAT catalog (Vn) and for SCAT catalog (Vns) for the period 1970 to 2017. The graph in Figure 4a is much smoother than in Figure 4b and contains 4 local extremes (1974, 1988, 1996 and 2006), which coincide with the corresponding local extremes in the graph from Figure 4b. The time series Vns correspond to the main tendencies of the $\mathrm{Vn}$ series, but it contains a large number of intermediate local extremes. 


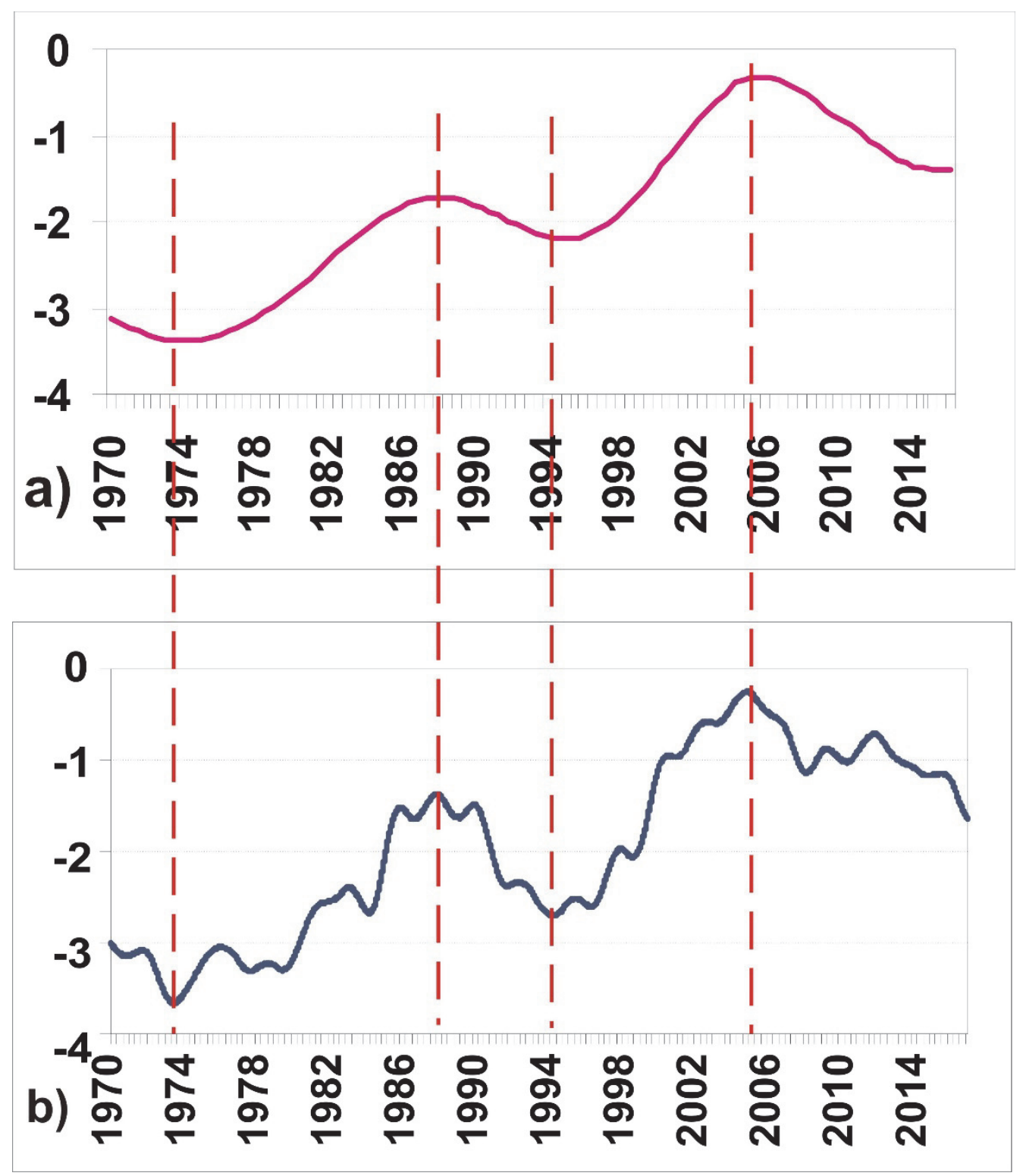

Figure 4. The low-frequency components of the AVER for the JCAT (Vn, fragment 4a) and for SCAT catalogs (Vns, fragment 4b) for the period 1970 to 2017.Red dotted lines correspond to local extremes of the Vn values.

A careful analysis of the dynamics of amplification and attenuation of the SA (Levin \&Sasorova, 2015a) and variation of the $\mathrm{Vn}$ value showed that the duration of the time interval between two neighboring local minima of the $\mathrm{Vn}$ values and the maximum values of the density of seismic events in time (Figure 3) does not remain constant. Therefore, attempts to find the main periodicity in the change of the SA in time were not productive today although they were undertaken repeatedly. It should be noted that the periodicity of about 30 years was indicated in a number of sources (Levin\&Sasorova, 2015b; Lutikov\&Rogozhin, 2014; Sasorova, Andreeva, \& Levin, 2013).

\section{Comparative Analysis of the Time Series}

A comparative analysis of the distribution of the density of seismic events over time and space and the low-frequency component of AVER is given below. Figure 5 and Figure6 show the two-dimensional seismic event density distributions (the right-hand parts of both figures) and the variation of the Vn value (left parts, respectively) for the two observation periods 1720-1890 and 1895- 2015 years. To analyze the distributions of seismic events in space, all EQ's were divided into 18 latitudinal belts (the size of each belt is $10^{\circ}$ ). 


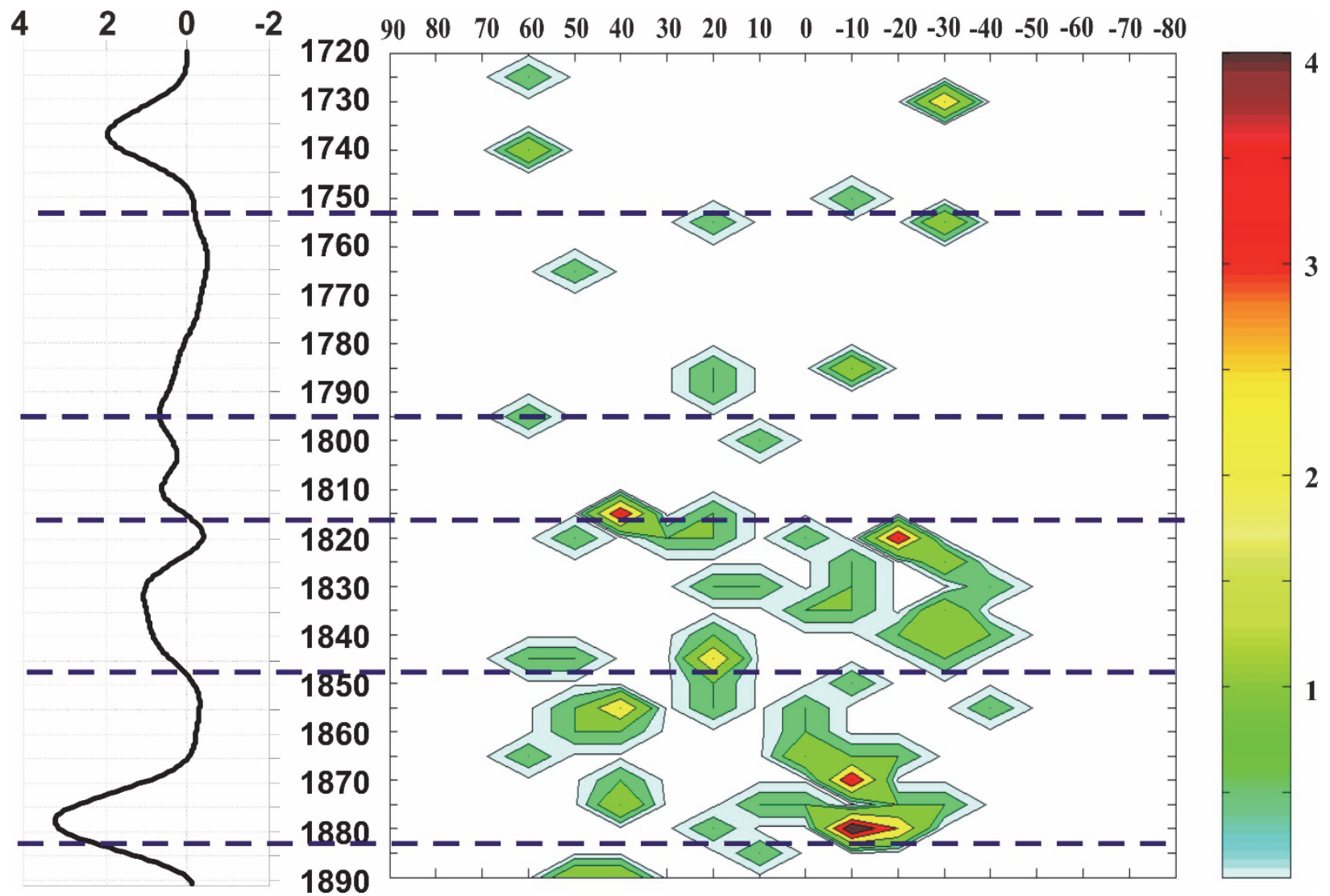

Figure 5. Two-dimensional EQ seismic event density distributions (the right-hand fragment) and variations in the Vn (left fragments) value for the period 1720-1895

Latitudinal belts are indicated on the right upper part of the horizontal axis of the two-dimensional distribution of density EQ's, and the values of Vn are on the upper part of the horizontal axis of the left fragment. The time scale, common for both fragments, is located in the middle; each number represents the upper boundary of the corresponding five-year interval. In the rightmost part of both figures is a color scale corresponding to the density of seismic events. The dashed lines intersecting both fragments of the figures correspond to the maxima of the seismic activity.

Two-dimensional distributions presented on the Figure 5-7 are show tendency to group events in time and in space. The current five-year interval from 2016 to 2020 is not included in the figures 6-7 due to incompleteness of data for this period.

The analysis shows that the stages of decreasing the angular velocity of rotation (braking) are accompanied by an increase in the density of seismic events, and the stages of increasing the angular velocity of rotation (acceleration) are accompanied by a decrease in the density of events. At the stages of deceleration the SA increases both in the Northern hemisphere of the Earth and in the Southern hemisphere.

In high latitudes SA is practically absent. The spatial peaks of the SA in the Northern Hemisphere are observed in latitudes $70^{\circ} \mathrm{N}-50^{\circ} \mathrm{N}$ and $50^{\circ} \mathrm{N}-30^{\circ} \mathrm{N}$. The most significant growth of SA was observed at the beginning of the 20th century in the period 1872-1905. At the intervals 1895-1915, increases in SA occur almost simultaneously, both in the latitude $70^{\circ} \mathrm{N}-50^{\circ} \mathrm{N}$ and latitudes $50^{\circ} \mathrm{N}-30^{\circ} \mathrm{N}$. After 1925 , during the last period of observation, the peaks of the SA in the Northern Hemisphere and in the Southern Hemisphere were displaced in time relative to each other. This is especially noticeable on the two-dimensional energy distribution. 


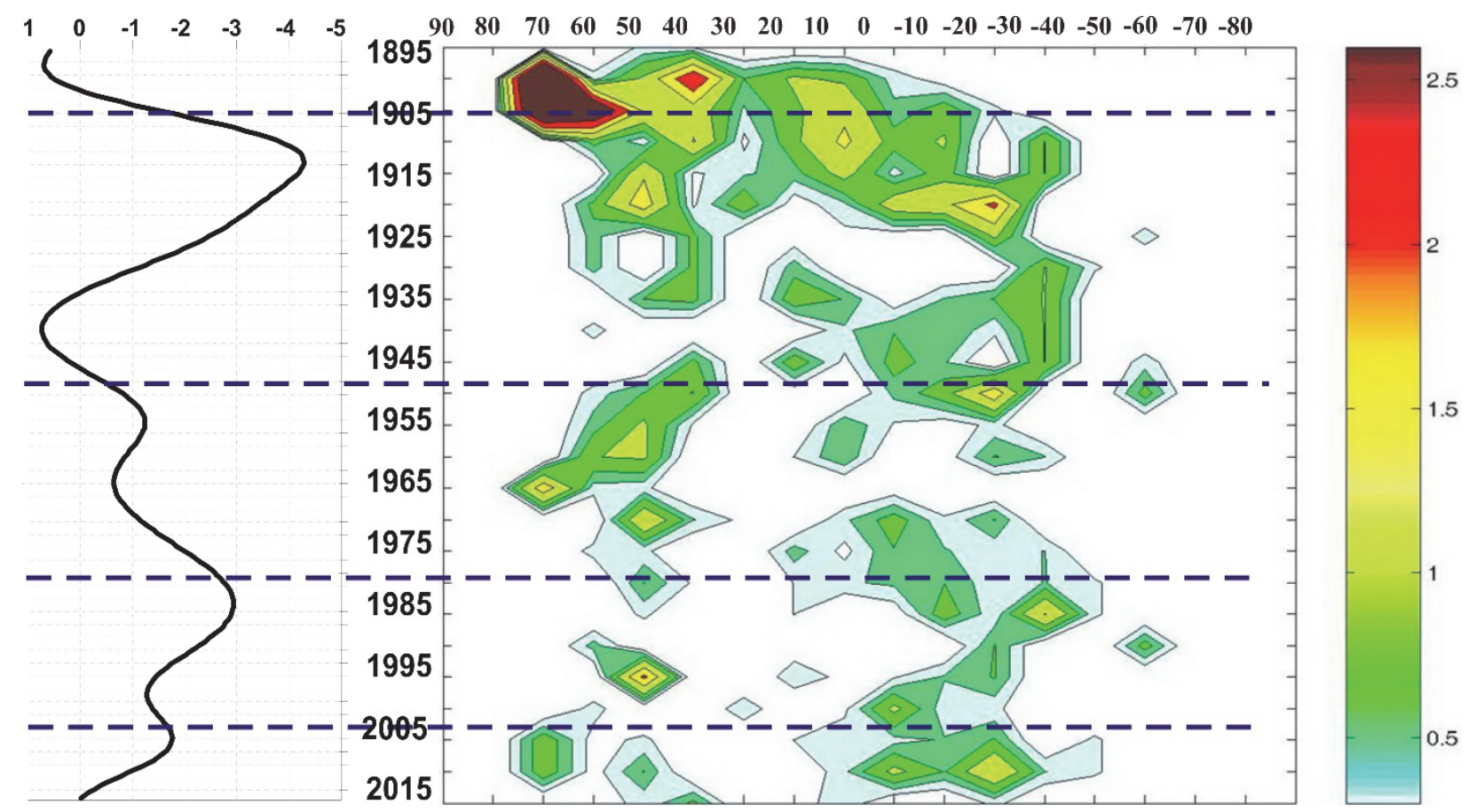

Figure 6. Two-dimensional EQ seismic event density distributions (the right-hand fragment) and variations in the Vn (left fragments) value for the period 1895-2015

Figure 7 shows the two-dimensional distribution of the energy released from earthquakes and variations in the value of $\mathrm{Vn}$ for the period 1895-2015. The notations are the same as in Figure 6.

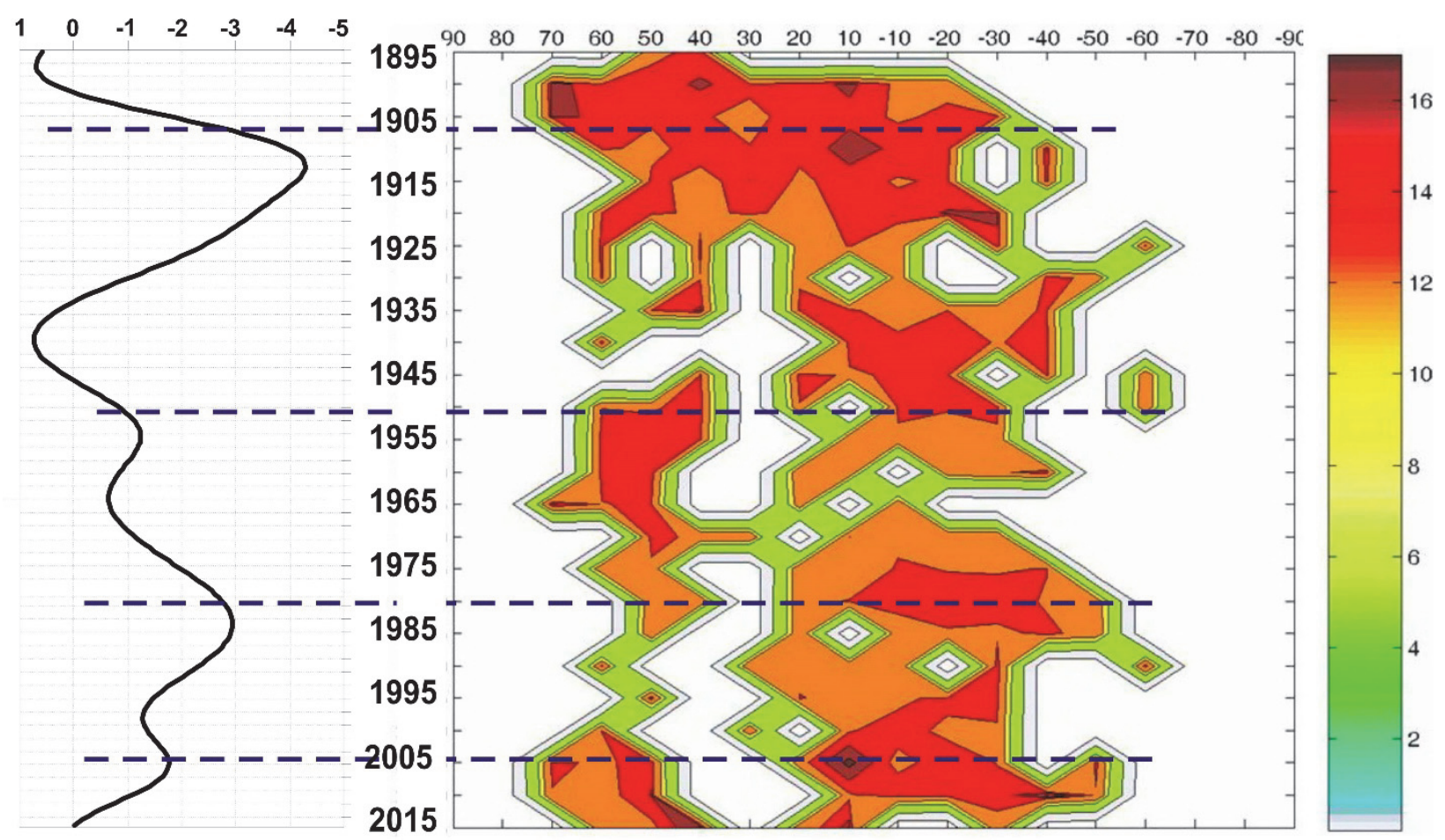

Figure 7. Two-dimensional distribution of the energy released from earthquakes (the right-hand fragment) and variations in the Vn value (left fragments) for the period 1895-2015 
For the low-frequency component of the AVER increment over the time interval $\Delta \mathrm{t}$ was determined as $-\Delta \mathrm{Vn} / \Delta \mathrm{t}$. This value is some estimate for accelerating the Earth's rotation. Positive values of the increments correspond to the stages of acceleration of the Earth, and negative values correspond to the stages of deceleration.

Two time series for each of the two observation periods 1720-1890 and 1895-2017 are shown in Figure 8 and Figure 9. The graph for the angular velocity increments is given in fragments a). The fragment $b$ ) of both figures shows the density distribution of strong EQ's (with $\mathrm{M} \geq 7.5$ ) over five-year intervals. The dotted vertical lines correspond to five-year intervals with the maximum SA.

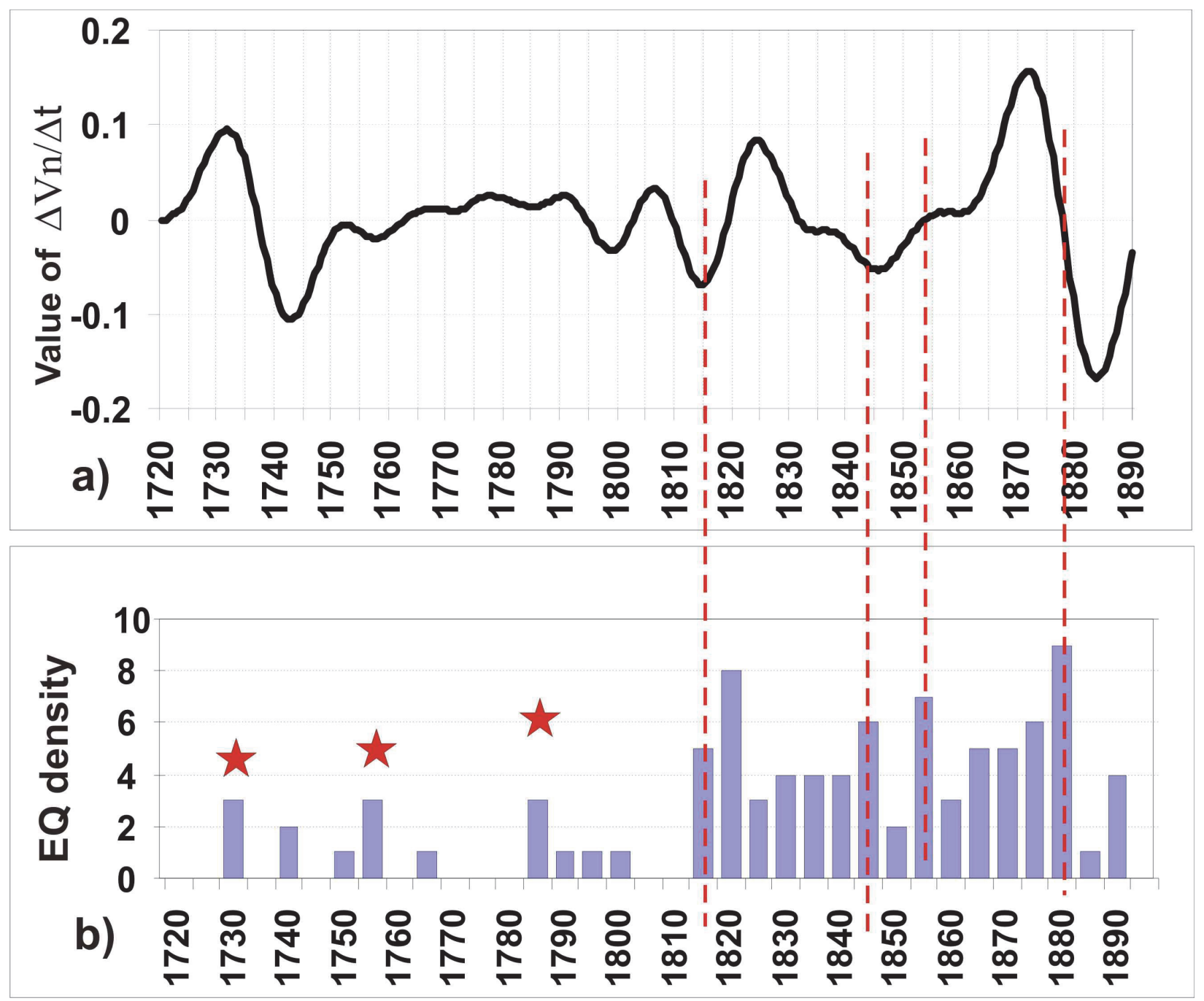

Figure 8. Comparative analysis for the period from 1720 to 1890 . a) - increment of the Vn value; b) - density distribution of strong earthquakes (with $\mathrm{M} \geq 7.5$ ) over five-year intervals. The vertical axes give the number of earthquakes in each five-year interval, horizontal axes - the upper boundaries of the five-year intervals (year)

Comparison of the time series of the increment of the low frequency component of the AVER $(\Delta \mathrm{Vn} / \Delta t)$ and the distributions of the EQ's density, shows that the maxima of the SA correspond to the final stages of the decreasing the angular velocity of rotation (i.e., braking stages).

The time intervals with the minimum of the SA correspond to the final stages of the increasing the angular velocity (acceleration stages). Thus it should be noted that the local minima of the angular velocity increments $(\Delta \mathrm{Vn} / \Delta \mathrm{t})$ practically coincide in time with the maxima of the SA. I.e. the maximum amplification of the SA occurs at the stage of maximum deceleration.

Earlier (Levin \&Sasorova, 2015a; Levin \&Sasorova, 2015b; Lutikov\&Rogozhin, 2014; Sasorova, Andreeva, \& Levin, 2013) it was revealed a fourfold increase in the density of seismic events in the five-year intervals of the 
first quarter of the 20th century (compared to the second half of the 20th century). It is this period (1895-1905) that corresponds to the prolonged and the most significant Earth's deceleration over the entire observation period from 1720 (a decrease in the values of the Vn, Figure 3).

An additional study showed that the position of the maxima and minima in the EQ distributions is retained when the five-year intervals for averaging the SA are replaced by three-year or six-year intervals.

From the second half of 2005 to the present, the decrease in the angular velocity of the Earth's rotation begins to be observed. The decrease in the values of $\mathrm{Vn}$ for this period can be observed on the right side in Figure 3 (indicated by the black thick dotted vertical line). Thus, at present the Earth enters the initial phase of the process of deceleration. According to our results, a new stage of deceleration can be characterized by an increase in the SA. Signs of the SA growth may begin to appear in the initial phase of deceleration.

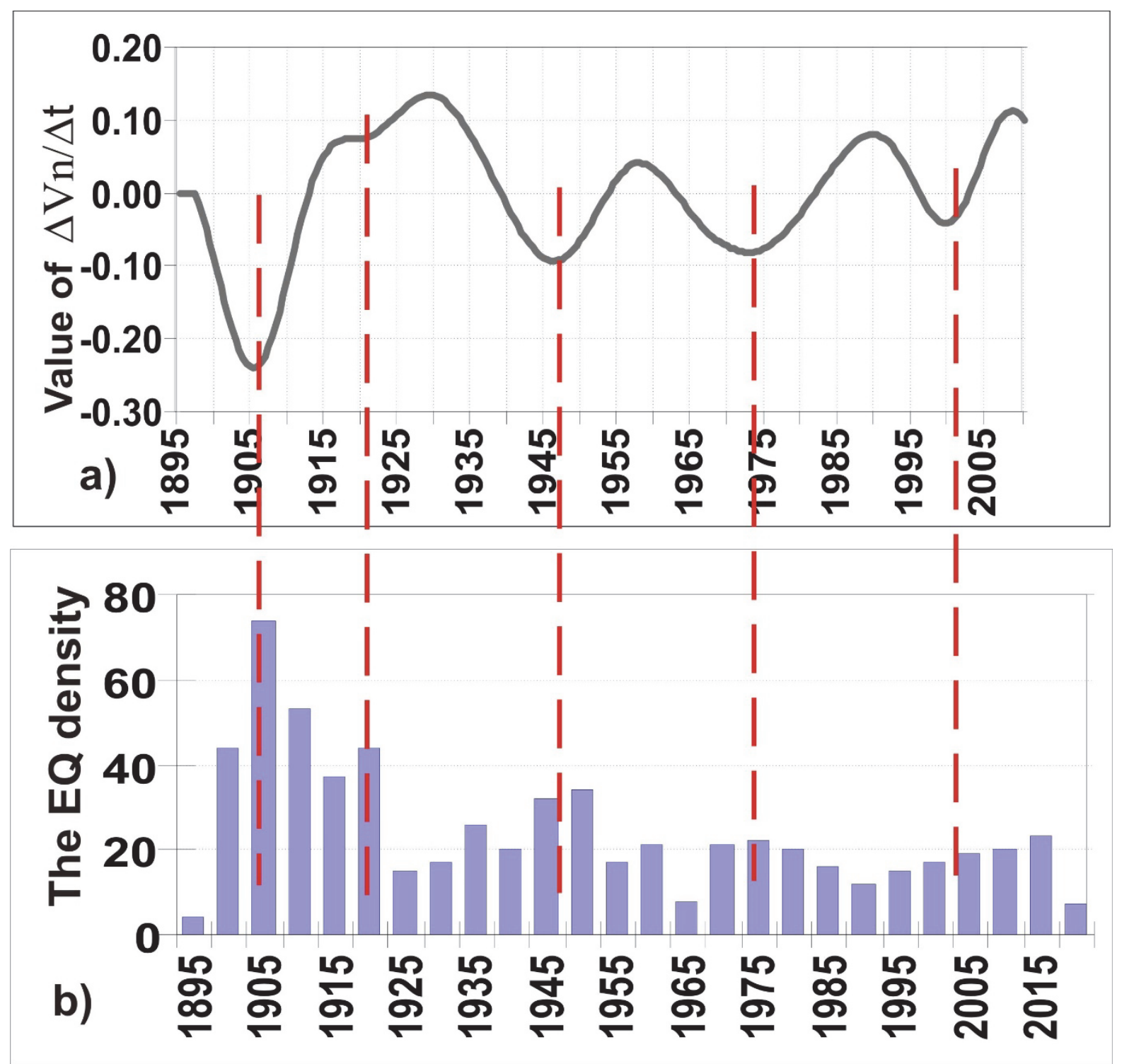

Figure 9. Comparative analysis for the period from 1895 to 2017.The notations are the same as in Figure 8.

We note the increase in destructive seismic events in Japan (Tohoku, 2011), a series of events in Italy since August 2016, a series of events in New Zealand (September, November 2016), in Japan (November 2016) and a number of other events. In 2017 and in early 2018 there were also 11 destructive earthquakes ( 7 of them in the equatorial zone and one in Alaska and the Aleutian Islands). 

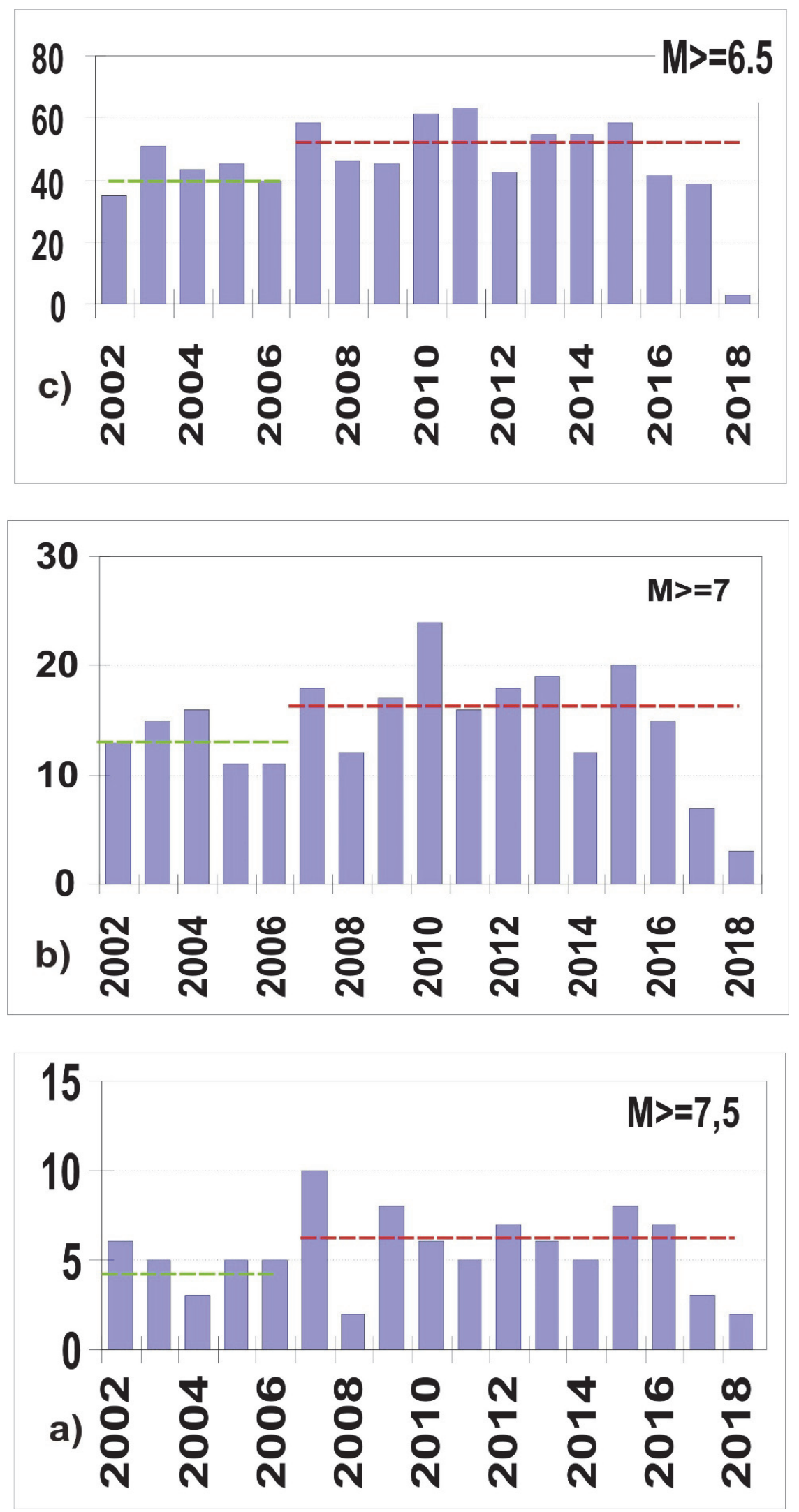

Figure 10. Green dashed horizontal lines correspond to the average number of events per year for the period from 1996 to 2006, and red dashed horizontal lines to the average number of events per year for the period from 2007 to 2018. The vertical axes give the number of earthquakes in each year interval, horizontal axes - the time scale (year). Magnitude is indicated in the upper right corner of each fragment 
Let us consider further how global seismic activity changed before and after 2006 (from 1996 to 2006 and from 2007 to 2018). The three fragments of Figure 10 show the distributions of the number of seismic events per one year for mentioned above periods for several magnitude ranges. It should be noted that for 2018 we had reliable data on seismic events only in January. Therefore, the number of events in 2018, shown in Figure 10, is very small.

Table 1. Presents the average values of the number of events per year (AV) and standard deviation (STDEV) for each magnitude range for both periods

\begin{tabular}{lcccccc}
\hline Magnitude range & \multicolumn{2}{c}{$\mathrm{M}>=7.5$ (93 events) } & \multicolumn{2}{c}{$\mathrm{M}>=7.0$ (181 events) } & \multicolumn{2}{c}{$\mathrm{M}>=6.5$ (765 events) } \\
\hline Observation. periods & $2000-2006$ & $2007-201$ & $2000-2006$ & $2007-201$ & $2000-2006$ & $2007-201$ \\
& & 7 & & 7 & & 7 \\
AV & 4.75 & 6.27 & 13.2 & 16.8 & 42.6 & 49.89 \\
STDEV & 1.1 & 2.3 & 2.28 & 4.6 & 5.93 & 7.81 \\
\hline
\end{tabular}

Let us denote the values of AV for the periods 2000-2006 and 2006-2017 as AV1 and AV2, and the values of STDEV for the same periods as STDEV1 and STDEV2.

Table 2. Present sthe percentages for AV2/AV1 and for STDEV1/STDEV2 for tree magnitude ranges.

\begin{tabular}{|c|c|c|c|}
\hline Magnitude range & $\mathrm{M}>=7.5$ & $\mathrm{M}>=7.0$ & $\mathrm{M}>=6.5$ \\
\hline percentages & $1.32 \%$ & $1.23 \%$ & $1.08 \%$ \\
\hline AV2/AV1 & & & \\
\hline percentages & $210 \%$ & $202 \%$ & $131 \%$ \\
\hline STDEV1/STDEV2 & & & \\
\hline
\end{tabular}

Analysis of the tables shows that the average number of events per year since 2007 is increasing. The most noticeable increase is observed for events with $\mathrm{M}>=7.5$. The STDEV value after 2006 for some magnitude ranges $(M>=7.5$, and $M>=7.0)$, more than doubles. Those scatter of seismic events over time in braking stage is greatly increased.

Analysis of the distributions of seismic events in the stage of deceleration shows the unevenness of the SA both in time and in space. In some years there is a sharp increase in the number of the EQs, and then a decrease may occur. And the EQ groups in the deceleration phase travel in time from one region of the Earth to another.

It should be noted that at present we are not yet able to determine how long and with what intensity the oncoming braking process will continue. Now it is currently unknown will this be an extended period of time when the Vn values sharply decreases as in 1872-1905, or a short period of decline in the Vn values and the increment values $(\Delta \mathrm{Vn} / \Delta \mathrm{t})$ that are insignificant in absolute value. Attempts to predict the behavior of the angular velocity of rotation $(v)$ in time in the scientific literature have not yet been revealed. Therefore, we can not predict the time when the maximum of the seismic activity will be reached. It was noted that the basic model of the authors (Levin \&Sasorova, 2015b) was confirmed in 2017 in (Bendick\&Bilham, 2017; Levin et al., 2017).

It is well known that any filtering (smoothing) does not give exact values for several initial and final values of the filtered time series due to the specifics of the filtering procedure itself. The larger is the averaging window, the more number of the incorrect values appear at the tail of the series. Therefore, for correct analysis of the trend of changing the low-frequency component of the AVER for edge values of time series (for period from 2006 to 2017), it is more informative to use the low-frequency component Vns (for the SCAT catalog) instead of Vn, for which the averaging window is significant larger than for the Vns. The edge values of Vns show a strong tendency to continue the cycle of reducing the angular velocity of rotation at present (left part of the Figure $4 \mathrm{~b}$ ) and, correspondingly, an increase in the seismic activity.

An estimate of the energy released into the Earth's lithosphere due to variations in the Earth's rotation velocity was made earlier by several authors. The energy of the celestial body as a result of the instability of rotation should increase as a result of variation of the angular velocity and the moment of inertia of the body: $\mathrm{dE} / \mathrm{E}=2$ $\mathrm{dv} / \mathrm{v}+\mathrm{dI} / \mathrm{I}$. It was shown in (Varga, Gambis, Bus, \&Bizouard, 2005) that $\mathrm{dE} \approx 10^{20} \mathrm{~J}$ and by $2-3$ orders of 
magnitude exceeds the total earthquake energy on Earth for the year $\left(\approx 10^{18} \mathrm{~J}\right)$. It was shown in (Levin, Domanski, \&Sasorova, 2014) that the most important is the increase in the angular velocity, then the estimate of the variation in the energy of the rotating body is $\mathrm{dE}=\mathrm{E} * \mathrm{dv} / \mathrm{v}=10^{29} * 10^{-8}=10^{21} \mathrm{~J}$. Thus, if there is a mechanism for transferring energy from the efficiency even less than $1 \%$, the change in the rotation velocity of the Earth can cause earthquakes.

Analysis of the physics of the connection between the deceleration and acceleration of the Earth's rotation and the increase or decrease of its SA in this paper was not considered.

However, it was shown earlier (Levin, Domanski, \&Sasorova, 2014) that an increase in the angular velocity of the Earth's rotation leads to an increase in the degree of compression of our planet, an increase in its equatorial radius, and an increase in its surface area, while maintaining the volume of the planet. Because of this, the stress between the lithospheric plates can decrease and, accordingly decrease the degree of the seismic activity.

While when the Earth's rotation velocity decreases, the opposite situation arises: the polar compression of the ellipsoid of rotation decreases, the surface area of the body is reduced, resulting in a deficit in the area of the shell of the solid crust. At the boundaries of tectonic plates deformation of compression develops; areas of local compressive stresses appear in the system. Zones with a highly non-equilibrium state of matter begins develop, which leads to the occurrence of earthquakes. It can be considered that further study of the relationship between changes in the Earth's rotation velocity and the activation of the SA will contribute to the development of a new approach to solving the problem of the causes of seismic activity growth and the occurrence of earthquakes.

\section{Acknowledgements}

This research was performed in the framework of FASO, Russia (0149-2018-0015) and supported in part by RFBR (13-05-00060, 16-05-00089) The authors consider it their pleasant duty to express gratitude to M.V. Rodkin and L.M. Bogomolov for the opportunity to discuss the physical ideas of the work and useful comments. The work was carried out at the Shirshov Institute of Oceanology of RAS, Moscow, Russia and at the Institute of Marine Geology and Geophysics, Far East Branch of the Russian Academy of Sciences, Yuzhno-Sakhalinsk, Russia.

\section{References}

Bendick, R., \& Bilham, R. (2017), Do weak global stresses synchronize earthquakes? Geophysical Research Letters, 44, 1-8. https://doi.org/10.1002/2017GL074934.

Historical Tsunami Database for the WorldOcean, from 2000 BC to Present. (2016), Tsunami Laboratory, ICMMG SD RAS, Novosibirsk. Retrieved from http://tsun.sscc.ru/nh/tsunami.php

International Earth Rotation and Reference System Service (IERS). Retrieved from http://iers.org

Levin, B. W., \& Sasorova, E. V. (2015a). Dynamics of Earth's seismic activity over 120 years. Doklady Earth Sciences, 461(1), 82-87. https://doi.org/10.1134/S1028334X15030034

Levin, B. W., \& Sasorova, E. V. (2015b). On the relationship between variations in the Earth's rotation velocity and its seismic activity. Doklady Earth Sciences, 464(1), 987-991. https://doi.org/10.7868/ S0869565215270183

Levin, B. W., Sasorova, E. V., Steblov, G. M., Domanskii, A. V., Prytkov, A. S., \& Tsyba, E. N. (2017). Variations of the Earth`s rotation rate and cyclic processes in geodynamics. Geodesy and Geodynamics, 8, $206-212$. http://dx.doi.org/10.1016/j.geog.2017.03.007

Levin, B., Domanski, A., \& Sasorova, E. (2014). Zonal concentration of some geophysical process intensity caused by tides and variations in the Earth's rotation velocity. Advances Geosciences, 35, 137-144. https://doi.org/10.5194/adgeo-35-137-2014

Lutikov, A. I., \& Rogozhin, E. A. (2014). Variations in the intensity of the global seismic process during the XX - early XXI centuries. Physics of the Earth, 50(4), 484-500. https://doi.org/10.1134/S1069351314040089

McCarthy, D. D., \& Babcock, A. K. (1986). The length of the day since 1656. Physics of the Earth and Planetary Interiors, 44, 281-292.

Morrison, L. V. (1973). Rotation of the Earth from A.D. 1663 - 1972 and the constancy of G. Nature, 241,519-520.

National Earthquake Information Center of the US Geological Survey (NEIC USGS): Retrieved from http://www.ncedc.org/anss/catalog-search.html 
Sasorova, E. V., Andreeva, M. Yu., \& Levin, B. W. (2013). Dynamics of seismicity of the Kuril island arc. Pacific Geology, 32(1), 75-84. https://doi.org/10.1134/S1819714013010077

Sidorenkov, N. S. (2002). Physics of Earth's rotational instabilities.Moscow, Science:Fizmatlit. (in Russian).

Varga, P., Gambis, D., Bus, Z., \& Bizouard, Ch. (2005). The relationship between the global seismicity and the rotation of the Earth. Journées 2004 - systèmes de référencespatio-temporels. Fundamental astronomy: new concepts and models for high accuracy observations. Paris, 20-22 September 2004, edited by N. Capitaine, Paris: Observatoire de Paris, ISBN 2-901057-51-9. P. 115-120.

\section{Copyrights}

Copyright for this article is retained by the author(s), with first publication rights granted to the journal.

This is an open-access article distributed under the terms and conditions of the Creative Commons Attribution license (http://creativecommons.org/licenses/by/4.0/). 